\title{
Coexistence of axial spondyloarthritis and thromboangiitis obliterans in a young woman
}

\author{
G. Lopalco ${ }^{1}$, F. lannone ${ }^{1}$, D. Rigante ${ }^{2}$, A. Vitale ${ }^{3}$, M.E. Mancini ${ }^{4}$, \\ M. Covelli', G. Lapadula ${ }^{1}$, L. Cantarini ${ }^{3}$ \\ ${ }^{1}$ Interdisciplinary Department of Medicine, Rheumatology Unit, Policlinico of Bari; \\ 2Institute of Pediatrics, Università Cattolica Sacro Cuore, Rome; \\ ${ }^{3}$ Research Center of Systemic Autoimmune and Autoinflammatory Diseases, Rheumatology Unit, \\ Policlinico Le Scotte, University of Siena, Siena; \\ ${ }^{4}$ Interdisciplinary Department of Medicine, Diagnostic Radiology Unit, Policlinico of Bari, Italy
}

\begin{abstract}
A peculiar coexistence of axial spondyloarthritis and ischemia of the feet and the fourth finger of the left hand in a young woman, who was a heavy smoker, is discussed in this report. This picture was considered within the context of thromboangiitis obliterans. Positivity of anti-nuclear antibodies and mild elevation of inflammatory parameters were noted. Computed tomography angiograms of upper and lower limbs showed luminal narrowing and occlusion of the left humeral, left anterior/posterior tibial and right anterior tibial arteries. Daily iloprost perfusions were started, and smoking cessation was strongly recommended. Coldness and rest pain in the distal extremities improved within a few weeks. The possibility that spondyloarthritis might precede the clinical picture of thromboangiitis obliterans should be considered in heavy smokers.
\end{abstract}

SUMMARY

Key words: Thromboangiitis obliterans, Axial spondyloarthritis.

Reumatismo, 2015; 67 (1): 17-20

\section{INTRODUCTION}

T hromboangiitis obliterans (TAO), also known as Buerger's disease, is a segmental vasculitis of small and mediumsized arteries causing obstruction of blood vessels (1). The average age of onset is around 40-45 years, and males are more frequently affected (2). Although its etiology is unknown, tobacco consumption plays a critical role in its pathogenesis (3-5). Clinically TAO is characterized by coldness and rest pain in the distal extremities, due to ischemic manifestations which might also lead to Raynaud's phenomenon, acrocyanosis, and necrosis of finger tips (6). To date, the cornerstone of management is smoking cessation, whereas other treatments aimed at improving the blood flow and dilating the blood vessels are less effective (7). We herein describe the case of a female patient presenting with an uncommon coexistence of axial spondyloarthritis ( $\mathrm{SpA}$ ) and TAO.

\section{CASE REPORT}

In March 2014, a 45-year-old female heavy smoker was hospitalized for clinical assessment of ischemia of feet and the fourth finger of the left hand. Her medical history started in 2008 with inflammatory polyarthralgia in the wrists, feet, elbows, and knees accompanied by inflammatory low-back pain. HLA-B35 was found and tenosynovitis in tendons of her hands was detected by ultrasonography. Finally, according to the Assessment of Spondyloarthritis International Society (ASAS) classification criteria, an axial SpA was diagnosed on the basis of the outcome of spine magnetic resonance imaging, which revealed a right sacroiliitis (unavailable images). Over the following 6 years she was treated with prednisone, salazopyrin and etanercept, reaching a clinical amelioration of joint symptoms. In August 2013 the patient presented Raynaud's phenom- $\overline{\text { Corresponding author: }}$ Luca Cantarini Rheumatology Unit Policlinico Le Scotte University of Siena Viale Bracci, 1 - 53100 Siena, Italy E-mail: cantariniluca@hotmail.com 
enon, involving her left hand and feet, and rest pain of the fourth fingertip combined with ischemic ulceration. When admitted to our Unit, laboratory investigations were as follows: C-reactive protein $10 \mathrm{mg} / \mathrm{L}$ [normal value (n.v.) $<3$ ] and erythrocyte sedimentation rate $31 \mathrm{~mm} / \mathrm{h}$ (n.v. <25). Autoimmunity tests revealed the positivity of anti-nuclear antibodies (titre of 1:640 with a fine speckled nuclear pattern), whereas anti-extractable nuclear antigens antibodies, anti-dsDNA antibodies, anti-citrullinated protein antibodies, rheumatoid factor, and anti-neutrophil cytoplasmic antibodies were all negative. Screening for hypercoagulopathy was also negative. Echocardiography and nailfold videocapillaroscopy gave normal results. However, an abnormal distal pulse was recognized at the left upper limb. As the patient complained low-back pain, X-ray of the pelvis was carried out, revealing the presence of subchondral sclerosis on the iliac side of the right sacroiliac joint. A computed tomography (CT)-angiography of upper and lower limbs was also performed, disclosing luminal narrowing and occlusion of the left humeral artery, left anterior and posterior tibial arteries and the right anterior tibial artery (Fig. 1). Moreover, CT scans showed erosions on the iliac side of the left sacroiliac joint (Fig. 2). On the basis of the narrowing and occlusion of arteries highlighted by CT-angiography, in agreement with the diagnostic criteria of Olin (8), the patient was diagnosed with TAO. Since the patient continued to complain inflammatory back pain, suggesting a loss of efficacy of etanercept, and considering that several urinary infections had occurred during the anti-tumor necrosis factor (TNF) treatment, etanercept was discontinued, while iloprost daily perfusions were started. Smoking cessation was strongly recommended.

Within a few weeks the patient reported a subjective improvement of coldness and rest pain: although the use of corticosteroids and sulfasalazine for the treatment of axial $\mathrm{SpA}$ is not supported by any evidence, the patient is currently treated with low-dose prednisone and salazopyrin, though a new biologic drug treatment has been proposed.
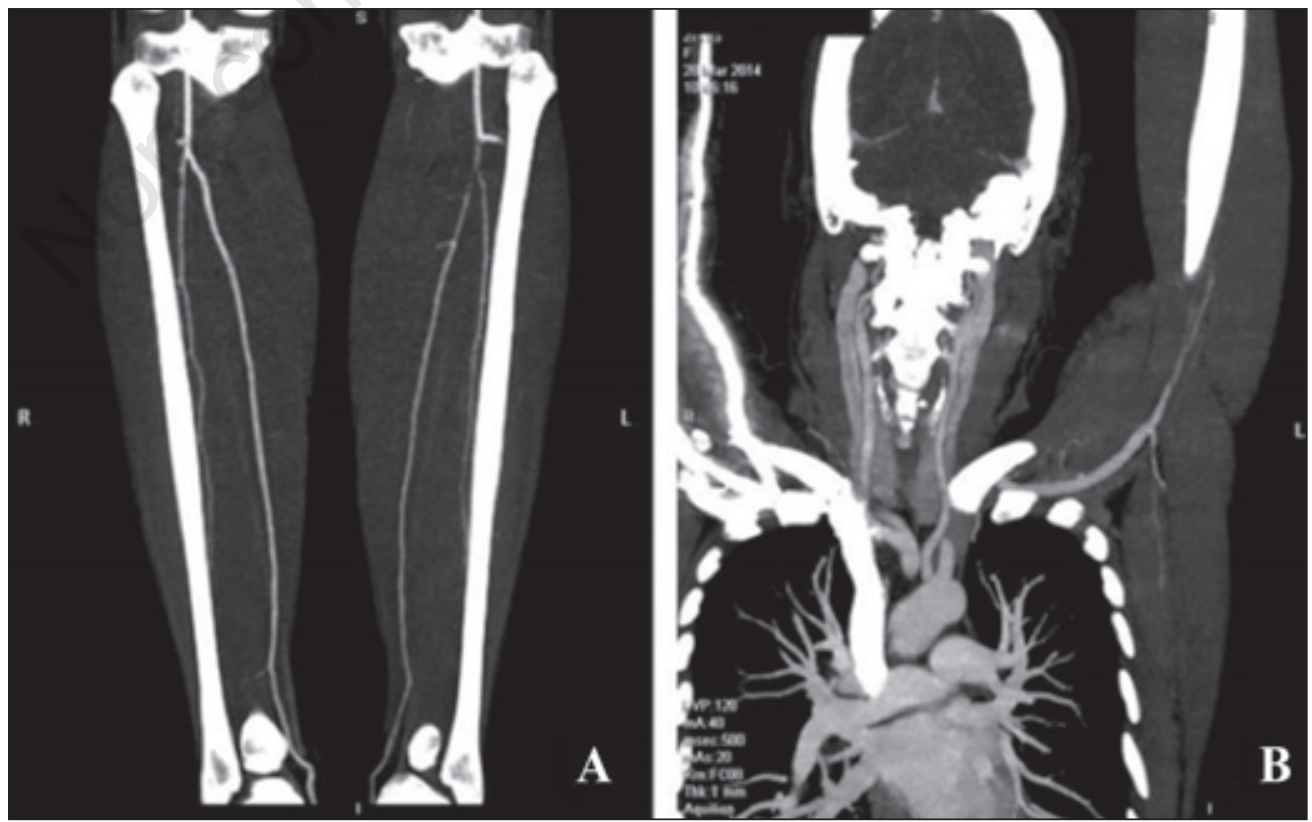

Figure 1 - Computed tomography angiograms showing luminal narrowing and occlusion of the left humeral artery (B), left anterior and posterior tibial arteries and right anterior tibial artery (A). 


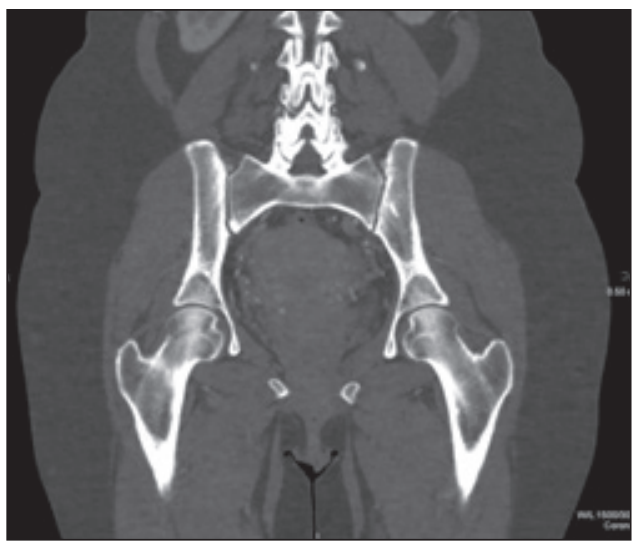

Figure 2 - Computed tomography scans displaying erosions on the iliac side of the left sacroiliac joint combined with a slightly poor definition of the iliac subchondral cortex.

\section{DISCUSSION}

TAO is a non-atherosclerotic vasculitis with a self-limiting course, characterized by inflammation of medium and smallsized arteries in the arms and legs, particularly radial and tibial arteries (9). Although its pathogenesis is not well understood, tobacco seems pivotal in the onset of this disease (2). In this regard, Papa et al. showed a cell-mediated immune response against human artery type-specific collagen in the presence of a peculiar genetic susceptibility (4). Women are less frequently involved by TAO, nevertheless a higher consumption of tobacco has been recently observed in the female sex (10). Keeping into account the age of onset, smoking habit, the type, size and location of vasculitis, we ruled out the possibility of other diagnostic explanations in our patient, such as Takayasu arteritis $(11,12)$. In agreement with the study of Sasaki et al., carried out to clarify the distribution of vasculitides in TAO, our patient presented vascular involvement of anterior and posterior tibial arteries in the lower extremities (9). Non erosive mono/oligoarthritis affecting large joints can occur up to 10 years before the first occlusive sign of TAO, often leading to a misinterpretation of the clinical phenotype (13). For these reasons, patients presenting with polyarthritis may be diagnosed with undifferentiated spondyloarthropathies, as occurred in our patient, showing signs suggestive of SpA six years before TAO (14). As suggested by Ernst et al., the possibility for a common inflammatory process to lead to both axial SpA and TAO could be the sole plausible explanation in our patient (15). Indeed the coexistence of $\mathrm{SpA}$ and leukocytoclastic vasculitis was reported previously $(16,17)$, corroborating the concept that vascular manifestations may be associated with inflammatory joint involvement. Regarding our patient, smoking might have played a pathogenic key-role in the onset of these two diseases. Smoking is indeed capable of activating inflammatory cells, such as dendritic cells and Th17 lymphocytes through the NF-kB pathway, in SpA. (18). Likewise, nicotine may induce the expression of unknown antigens in the vascular endothelium, triggering immune-mediated responses that lead to the vascular damage involved in TAO (19).

\section{CONCLUSIONS}

The main feature of our case is the unreported association between axial SpA and TAO: although it is unclear if sacroiliitis reflects a mere association with TAO or if they represent two coinciding immunemediated disorders, the possibility that sacroiliitis might occasionally precede TAO should be contemplated in heavy smokers.

Conflict of interests: the authors declare no potential conflict of interest.

Contributions: GL and FI contributed equally to this manuscript.

\section{REFERENCES}

1. Dargon PT, Landry GJ. Buerger's disease. Ann Vasc Surg. 2012; 26: 871-80.

2. Arkkila PE. Thromboangiitis obliterans (Buerger's disease). Orphanet J Rare Dis. 2006; 1: 14.

3. Adar R, Papa MZ, Halpern Z, Mozes M, Shoshan S, Sofer B, et al. Cellular sensitivity to collagen in thromboangiitis obliterans. $\mathrm{N}$ Engl J Med. 1983; 308: 1113-6. 
4. Papa M, Bass A, Adar R, Halperin Z, Schneiderman J, Becker CG, et al. Autoimmune mechanisms in thromboangiitis obliterans (Buerger's disease): the role of tobacco antigen and the major histocompatibility complex. Surgery. 1992; 111: 527-31.

5. Matsushita M, Shionoya S, Matsumoto T. Urinary cotinine measurement in patients with Buerger's disease - Effects of active and passive smoking on the disease process. J Vasc Surg. 1991; 14: 53-8.

6. Vijayakumar A, Tiwari R, Kumar Prabhuswamy V. Thromboangiitis obliterans (Buerger's disease) - Current practices. Int J Inflam. 2013; 2013: 156905.

7. Puéchal X, Fiessinger JN. Thromboangiitis obliterans or Buerger's disease: challenges for the rheumatologist. Rheumatology (Oxford). 2007; 46: 192-9.

8. Olin JW. Thromboangiitis obliterans (Buerger's disease). N Engl J Med. 2000; 343: 864-9.

9. Sasaki S, Sakuma M, Kunihara T, Yasuda K. Distribution of arterial involvement in thromboangiitis obliterans (Buerger's disease): results of a study conducted by the Intractable Vasculitis Syndromes Research Group in Japan. Surg Today. 2000; 30: 600-5.

10. Mills JL, Taylor LM Jr, Porter JM. Buerger's disease in the modern era. Am J Surg. 1987; 154: 123-9.

11. Ishikawa K. Diagnostic approach and proposed criteria for the clinical diagnosis of Takayasu's arteriopathy. J Am Coll Cardiol. 1988; 12: 964-72.
12. Arend WP, Michel BA, Bloch DA, Hunder GG, Calabrese LH, Edworthy SM, et al. The American College of Rheumatology 1990 criteria for the classification of Takayasu arteritis. Arthritis Rheum. 1990; 33: 1129-34.

13. Lambotte $O$, Chazerain $P$, Vinciguerra $C$, Meyer O, Ziza JM. Thromboangiitis obliterans with inaugural rheumatic manifestations. A report of three cases. Rev Rhum Engl Ed. 1997; 64: 334-8.

14. Puéchal X, Fiessinger JN, Kahan A, Menkès CJ. Rheumatic manifestations in patients with thromboangiitis obliterans (Buerger's disease). J Rheumatol. 1999; 26: 1764-8.

15. Ernst D, Baerlecken N, Schmidt R, Witte T. Large vessel vasculitis and spondyloarthritis: coincidence or associated diseases? Scand J Rheumatol. 2014; 43: 246-8.

16. Kobak S, Yilmaz H, Karaarslan A, Yalcin M. Leukocytoclastic vasculitis in a patient with ankylosing spondylitis. Case Rep Rheumatol. 2014; 2014: 653837.

17. Hsu CM, Kuo SY, Chu SJ, Shih TY, Chen A, Huang GS, et al. Coexisting IgA nephropathy and leukocytoclastic cutaneous vasculitis associated with ankylosing spondylitis: a case report. Zhonghua Yi Xue Za Zhi (Taipei). 1995; 55: 83-8.

18. Wendling D. IL-23 and IL-17 in ankylosing spondylitis. Rheumatol Int. 2010; 30: 1547.

19. Little MA, Savage CO. The role of the endothelium in systemic small vessel vasculitis. Clin Exp Rheumatol. 2008; 26: S135-40. 